\title{
Peptide drugs for photopharmacology: how much of a safety advantage can be gained by photocontrol?
}

\author{
Oleg Babii ${ }^{1}$, Sergii Afonin ${ }^{1}$, Tim Schober², Liudmyla V Garmanchuk², Liudmyla I \\ Ostapchenko ${ }^{3}$, Volodymyr Yurchenko ${ }^{4}$, Sergey Zozulya ${ }^{4}$, Oleksandr Tarasov, Iryna Pishel ${ }^{4}$, \\ Anne S Ulrich ${ }^{1,2}$ \& Igor V Komarov*,4,5,6 \\ ${ }^{1}$ Karlsruhe Institute of Technology (KIT), Institute of Biological Interfaces (IBG-2), POB 3640, 76021 Karlsruhe, Germany \\ ${ }^{2}$ KIT, Institute of Organic Chemistry (IOC), Fritz-Haber-Weg 6, 76131 Karlsruhe, Germany \\ ${ }^{3}$ Taras Shevchenko National University of Kyiv (TSNUK), Institute of Biology \& Medicine, prosp. Hlushkova 2, 03022 Kyiv, Ukraine \\ ${ }^{4}$ Enamine Ltd, vul. Chervonotkatska 78, 02066 Kyiv, Ukraine \\ ${ }^{5}$ TSNUK, Institute of High Technologies, vul. Volodymyrska 60, 01601 Kyiv, Ukraine \\ ${ }^{6}$ Lumobiotics GmbH, Auerstraße 2, 76227 Karlsruhe, Germany \\ *Author for correspondence: Tel.: +380 675001 151; igor.komarov@lumobiotics.com
}

\begin{abstract}
Aim: To verify whether photocontrol of biological activity could augment safety of a chemotherapeutic agent. Materials \& methods: $\mathrm{LD}_{50}$ values for gramicidin $\mathrm{S}$ and photoisomeric forms of its photoswitchable diarylethene-containing analogs were determined using mice. The results were compared with data obtained from cell viability measurements taken for the same compounds. Absorption, Distribution, Metabolism, and Elimination (ADME) tests using a murine cancer model were conducted to get insight into the underlying reasons for the observed in vivo toxicity. Results: While in vitro cytotoxicity values of the photoisomers differed substantially, the differences in the observed $\mathrm{LD}_{50}$ values were less pronounced due to unfavorable pharmacokinetic parameters. Conclusion: Despite unfavorable pharmacokinetic properties as in the representative case studied here, there is an overall advantage to be gained in the safety profile of a chemotherapeutic agent via photocontrol. Nevertheless, optimization of the pharmacokinetic parameters of photoisomers is an important issue to be addressed during the development of photopharmacological drugs.
\end{abstract}

Lay abstract: To critically assess the photopharmacological impact at the cellular and organismal level, systematic toxicity studies were conducted on representative types of photoswitchable drug candidates and their nonphotoswitchable counterparts. We analyzed the in vivo toxicity profiles of photocontrollable peptide derivatives of the natural antibiotic gramicidin $\mathrm{S}$ by comparing the effects of their two photoisomeric forms in mice. The $\mathrm{LD}_{50}$ values of the photoisomers were optimized to differ substantially. Hence, it was concluded that the maximum tolerated dose of the less-toxic isomer could be much higher than that of the parent compound, gramicidin S. This result justifies the use of peptidic photoswitchable compounds for future medical applications and encourages further efforts toward their development.

First draft submitted: 17 November 2019; Accepted for publication: 17 January 2020; Published online: 6 February 2020

Keywords: diarylethenes $\bullet$ in vivo toxicity $\bullet$ peptides $\bullet$ peptidomimetics $\bullet$ photocontrollable compound safety $\bullet$ photopharmacology $\bullet$ photoswitchable compounds

Photopharmacology is a rapidly expanding and highly innovative area of research focused on the development and application of photocontrollable compounds for therapeutic purposes. Even though the idea of using such compounds for the treatment of human diseases has been around for many decades, recent discoveries and the emergence of enabling technologies have transformed photocontrollable compounds into viable candidates as therapeutic agents in a clinical setting [1-4]. Initially, the term 'photopharmacology' referred to the use of photoreactive species as therapeutic agents [5], but that definition has been expanded to take into account current trends in this field. One such area of interest is the use of photoswitchable compounds for medical purposes, as 
these compounds contain photoisomerizable fragments (molecular photoswitches) that can exist in two or more isomeric forms that are interconvertible by light.

Two main strategies for the application of photoswitchable compounds in medicine are currently being pursued. The first strategy is centered on the use of photoswitchable molecules as surrogates or modulators for endogenous photosensitive compounds. Here, degenerative diseases, such as those related to vision or circadian rhythm disorders, can be treated through the use of photopharmacological agents. Photosensitive drug candidates aimed at vision restoration are very close to clinical applications [6,7]. The second, more popular strategy relies on the use of photocontrollable drugs in the same way as most nonphotocontrollable drugs, in other words, using them as directly acting at biological targets involved in pathological processes. A priori, it is believed that tolerability and safety parameters of the latter strategy should inherently be high due to photocontrol $[1,3]$; it is based on the concept that a photocontrollable drug can be administered in the inactive, less-toxic isomeric form which can then be activated by light with a high level of spatiotemporal precision at the desired site of action [8]. Light-induced or thermal inactivation of the photocontrollable drugs after they have exerted their desired action has also been postulated as a contribution to drug safety for patients and the environment as a whole [9].

Although the enhanced safety of photopharmacological drugs seems to be reasonably justified in theory, it has never been properly verified in mammals in practice. Numerous in vitro studies have already demonstrated that the isomers of a photoswitch-modified compound have considerably different affinities to their molecular targets in the human body and that the function of these targets could be efficiently photomodulated $[10,11]$. In many in vitro studies on cell lines, photoswitchable compounds have been shown to be practically inactive in one photoisomeric form but significantly active in the other $[4,12,13]$. This, however, is not proof of enhanced safety as it relates to photocontrol. The question of how much of a safety advantage can be gained in practice needs to be answered by carrying out a detailed toxicological profile for these drug candidates in animal models and, eventually, in clinical studies conducted with human subjects. Here, we report our attempt to address this question using rodent test subjects and representative types of photocontrollable biologically active compounds that are analogs of a chemotherapeutic agent known for its considerable in vivo toxicity.

The toxicity of drug candidates is a major concern in pharmacology since the adverse side effects often observed are the foremost cause of high attrition rates for investigational drugs [14]. According to an analysis conducted in 2012 [15], 36\% of all drug candidates entering pivotal clinical trials failed due to issues on clinical safety. Safety-related drug development failures highlight the importance of preclinical toxicology studies. Although there is growing concern about the use of animal models to predict human drug toxicity [16], numerous studies have revealed that rodent and nonrodent safety data are sufficiently predictive of the extent and type of adverse effects that could be experienced in subsequent human clinical trials [17-19], provided that the methodology of the preclinical safety data acquisition was correct [20]. Surprisingly, literature reports on the toxicity of photocontrollable biologically active compounds in multicellular organisms are very scarce, with most simply using lower vertebrates, such as zebrafish embryos, as their test subjects [21-25]. To the best of our knowledge, toxicity data on different photoforms of photoswitch-modified compounds have never been determined and compared in mammals [25-28].

Most of the studies on bioactive photocontrollable compounds published to date were designed using nonphotoisomerizable biologically active molecules as prototypes. These prototype molecules were modified with photoswitches to enable parent activity photomodulation. In a typical approach referred to as 'azologization' [27,28], a photoisomerizable azobenzene fragment is incorporated into the molecules of known biologically active compounds. Similar approaches have been reported for alternative types of photoswitches, including diarylethenes [29], spiropyranes [30], indigoid photoswitches [31] and others [32]. To assess the true gain in safety brought about by in vivo photocontrol, one must compare the efficacy and toxicity of the prototypes with those of the photoisomeric forms of their photoswitch-modified analogs. Lower toxicity for one photoisomer and a similar efficacy results for the other isomer(s), when compared with the prototype, would justify the use of that particular photocontrollable compound in human trials. Moreover, any known drug whose development was discontinued for reasons of insufficient safety parameters may have a second chance at being reconsidered and used as a prototype for the design of a safer photocontrollable analog. Therefore, we decided to evaluate the safety profiles of representative photocontrollable bioactive compounds and compare them with their prototype in this study.

\section{Materials \& methods}

Photocontrollable biologically active compounds

In this study, diarylethene-derived analogs of a cyclic decapeptide, the natural antibiotic gramicidin S (GS; Figure 1), 


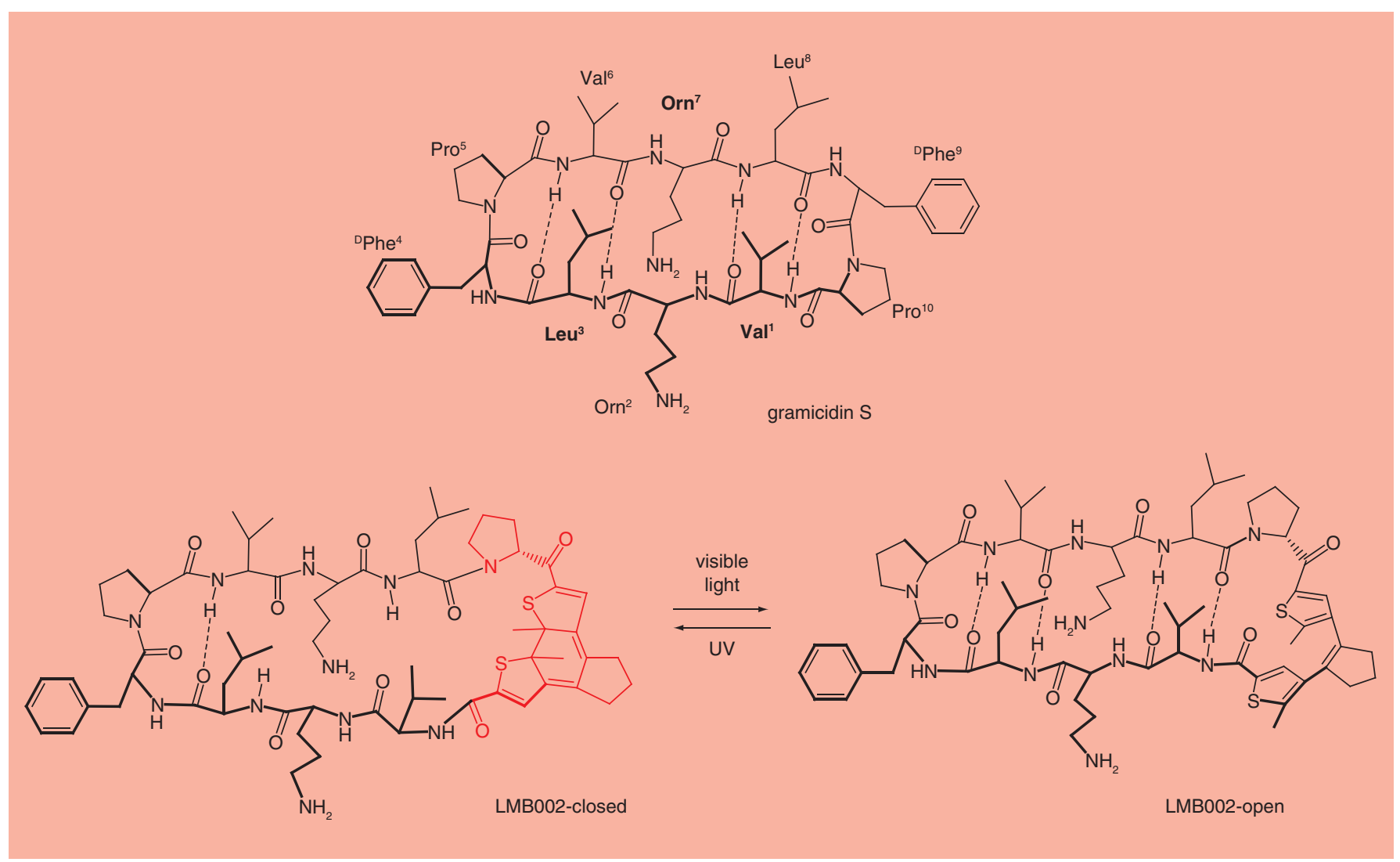

Figure 1. The prototype peptide, gramicidin S and its photocontrollable analog LMB002. The 'closed' diarylethene-based photoswitch is highlighted in color.

were used. GS has been known since the beginning of the antibiotic era [33]. The promising photocontrollable antimicrobial and anticancer activities of one of these GS analogs, LMB002, are thoroughly documented [12,13]. Four other derivatives, whose formulas are not disclosed here (LMB033, LMB037, LMB039 and LMB040), are single-point mutants of LMB002 with amino acid substitutions that gently modulate the polarity of the compound. The resulting diarylethene-containing cyclic peptidomimetics are of comparable molecular weight and solubility and all can exist in two thermally stable forms that have been designated as 'open' and 'closed'. Reversible interconversion between these forms can be achieved via UV $(\lambda=325 \mathrm{~nm})$ or red $(\lambda=653 \mathrm{~nm})$ light irradiation, as illustrated in Figure 1 for LMB002.

Peptide drugs are currently on the rise in the pharmaceutical industry [34] and photocontrollable peptides in particular are gaining momentum as a top priority lead in medicinal chemistry research [35]. Our prototype peptide, GS, is highly toxic when administered systemically $[33,36]$. Therefore, although GS is well known and has been used as a drug for more than 75 years, its medicinal use is restricted to topical applications due to its toxicity profile. Only one other paper demonstrated the anticancer effects exerted by this peptide in a murine tumor allograft model [37]. High mortality rates (between 60 and $80 \%$ ) have been attributed to the associated side effects. This inherent toxicity is the reason why GS was considered as an ideal prototype for the present study.

\section{Chemical synthesis \& characterization of the compounds}

All compounds studied in this work were prepared using the procedures described in [13]. Their purity, as determined by HPLC (see HPLC traces in the Supplementary Material), was higher than $96 \%$. All solutions of the photoisomerizable compounds for the cell assays and animal studies were prepared immediately before use and handled under reduced light in order to prevent uncontrolled photoisomerization. HPLC analysis of peptide solutions after conducting animal studies showed insignificant spontaneous photoswitching $(<3 \%)$.

Preliminary evaluation of the solubility of our compounds and selection of their appropriate formulation has been first carried out on 12 different formulations (see Supplementary Material for details). Cremophor EL-physiological 
saline (5\%:95\%) was identified as the best vehicle formulation, which provided the most stable suspensions or solutions of the tested compounds. Therefore, this formulation was used in all our in vivo studies.

Cell viability assay

All materials were from Sigma-Aldrich (MO, USA) or Biopharma (Kyiv, Ukraine). The cell lines Hep G2 (human hepatocyte carcinoma, ECACC 85011430), MCF-7 (human breast adenocarcinoma, ECACC 86012803), MIA-PaCa-2 (human pancreatic carcinoma, ECACC 85062806), MOR (human lung adenocarcinoma, ECACC 84112312), MOR/0.2R (human lung adenocarcinoma, doxorubicin-resistant, ECACC 96042335), COLO 205 (human colon adenocarcinoma, ECACC 87061208), H69 (human lung small cell carcinoma, ECACC 91091802), HEK 293 (human embryo kidney cells, ECACC 85120602), CHO (Chinese hamster ovary cells, ECACC 85050302) and HeLa (human cervix epithelioid carcinoma, ECACC 93021013) were originally from the European Collection of Authenticated Cell Cultures. Human malignant melanoma cell line WM 1158 and Lewis lung carcinoma (LLC) cells were obtained from the National Bank of Cell Lines and Tumor Strains at RE Kavetsky Institute of Experimental Pathology, Oncology and Radiobiology, National Academy of Sciences of Ukraine (Kyiv, Ukraine).

The cytotoxicity of the photocontrollable compounds in both photoforms was evaluated with a standard colorimetric assay using MTT (3-[4,5-dimethylthiazol-2-yl]-2,5-diphenyltetrazolium bromide). Cell growth inhibition was characterized by conventional $\mathrm{IC}_{50}$ values $[38,39]$ determined in a 96-well microtiter plate setup. As a culture medium, Dulbecco's modified Eagle medium supplemented with 10\% fetal bovine serum, $2 \mathrm{mM}$ L-glutamine and $40 \mu \mathrm{g} / \mathrm{ml}$ gentamicin was used. Cells were plated with a starting concentration of about $5 \times 10^{4} \mathrm{cells} / \mathrm{ml}(100 \mu \mathrm{l}$ per well). After a $24 \mathrm{~h}$ period of adaptation $\left(5 \% \mathrm{CO}_{2}, 100 \%\right.$ humidity, $\left.37^{\circ} \mathrm{C}\right)$, the tested compounds were added at different concentrations (in $100 \mu \mathrm{l}$ of Dulbecco's modified Eagle medium) to each well, except for the control, and the plates were incubated for additional $24 \mathrm{~h}$. Next, $10 \mu \mathrm{l}$ of the $12 \mathrm{mM}$ MTT solution was added to each well and the plates were left for $4 \mathrm{~h}$ incubation. Subsequently, the medium from each well was removed and $100 \mu \mathrm{l}$ of DMSO was added instead. The amount of formazan was determined from the optical density measured at $570 \mathrm{~nm}$. Four individual repetitions and ten different dilutions (twofold dilution series) were used for each tested peptide (starting at 256 and $512 \mu \mathrm{g} / \mathrm{ml}$ for open and closed forms, respectively). All experiments with the closed isomers (visible light-sensitive forms) were performed under reduced light to prevent uncontrolled photoisomerization. $\mathrm{IC}_{50}$ values were extrapolated from the viability-concentration plots (see Supplementary Material).

\section{Hemolytic activity assay}

To test the hemolytic activity of compounds, human erythrocytes (red blood cells [RBC]) were used. Citrate phosphate dextrose-stabilized blood bags with RBC suspensions of healthy donors were obtained from Karlsruhe Municipal Hospital (Karlsruhe, Germany). Two $172 \mathrm{mM}$ Tris-HCl buffers with the isotonic osmolarity of human blood $(310 \mathrm{mOs} / \mathrm{ml}), \mathrm{HB} 1$ and $\mathrm{HB} 2$ were used in the assay $\left(\mathrm{HB} 1 \mathrm{had} \mathrm{pH} 7.6\right.$ at $4^{\circ} \mathrm{C}$; $\mathrm{HB} 2$ had $\mathrm{pH} 7.6$ at $37^{\circ} \mathrm{C}$ ). The RBCs were washed twice in $\mathrm{HB} 1$ by centrifugation at $1700 \mathrm{rpm}, 4^{\circ} \mathrm{C}$ and diluted to $0.5 \%$ hematocrit with HB2. Compounds were dissolved in $40 \mu \mathrm{l}$ of DMSO and diluted to $400 \mu \mathrm{l}$ by HB2. The starting solutions were used to prepare twofold dilution series of $200 \mu \mathrm{l}$ aliquots in $1.5 \mathrm{ml}$ microcentrifuge tubes. Two independent series of seven dilutions were prepared (starting at 64 or $256 \mu \mathrm{g} / \mathrm{ml}$ for open and closed forms, respectively). The hemolysis process was initiated by the addition of $200 \mu \mathrm{l}$ of the RBC suspension to the peptides in HB2. The samples were incubated under periodic agitation for $30 \mathrm{~min}$ at $37^{\circ} \mathrm{C}$; their content was pelleted for $10 \mathrm{~min}$ at $13000 \mathrm{rpm}$. The absorption of the supernatant at $540 \mathrm{~nm}$ gave the extent of hemolysis, with $0 \%$ measured from the peptide-free negative control and $100 \%$ from the treatment with $0.1 \%$ Triton X-100 (positive control). To ensure that the colorimetric readout does not interfere with the absorption changes of the diarylethene fragments, the peptides in the closed forms were converted to their open colorless forms before the absorption measurement (by a brief sample exposure to visible light). Fifty percent hemolysis $\left(\mathrm{HC}_{50}\right)$ values were extrapolated from the hemolysis-concentration plots (see Supplementary Material).

\section{In vivo experiments}

All in vivo experiments were performed at the facilities and under the ethics guidelines of Enamine/Bienta (both Kyiv, Ukraine) [40]. 


\section{Bioethics}

Animal care and experimental procedures were performed following the 8th Edition (2011) of the Guide for the Care and Use of Laboratory Animals by the National Research Council (DC, USA) and under Article 6 of the Law of Ukraine 'On Medicines'. This study was approved by the Bioethics Commission of Educational and Scientific Centre 'Institute of Biology and Medicine' of the Taras Shevchenko National University of Kyiv (Ukraine). Minimum possible numbers of animals were used in the studies and literature data were used wherever available.

\section{Animals}

Balb/c or C57BL/6 female mice, 9-10 weeks old, 18.3-27.5 g bodyweight were used for the studies (average bodyweight across all experimental groups was $21.9 \mathrm{~g}$ ). Balb/c line was used in phototoxicity studies [41]. C57BL/6 were used in acute toxicity and pharmacokinetic studies. During the 7-day acclimatization period, three to six animals were kept in each cage. All animals were under observation; only animals free from any symptoms of sickness were used. Animals were randomly assigned to experimental groups. Each cage contained animals from a uniform group. Except for the light regimen (see below), the housing conditions of the animals were the standard, accepted for such studies [42].

\section{Acute toxicity evaluation of LMB002, LMB033, LMB037, LMB039, LMB040}

The animals were divided into groups of five to six individuals. One group of five vehicle-dosed animals was used as a control. All animals were kept under reduced light conditions during the intravenous dosing and in complete darkness after the administration of compounds. Observations for mortality and signs of toxicity were done under reduced light at $30 \mathrm{~min}, 1 \mathrm{~h}$ and $2 \mathrm{~h}$ after administration and, after that, daily for 14 days. Bodyweights measurements and calculation of mean bodyweight/group were performed daily. Formulations were prepared under reduced light before the administration and were freshly sterile-filtered to avoid inadvertent infection. Tested compounds were administered in a volume corresponding to $10 \mathrm{ml} / \mathrm{kg}$ of the bodyweight. All animals fasted for $3 \mathrm{~h}$ before dosing. The toxic effects were assessed by visual observation. The state of skin and fur, eyes and mucous membranes, respiratory system, blood circulation, peripheral and central nervous systems and changes in locomotor activity were all examined. The occurrence of tremors, seizures, salivation, diarrhea or apathy was monitored. Euthanasia was performed following the Organisation for Economic Cooperation and Development (Paris, France) recommendations [42]. Gross pathology assessment was carried out according to Enamine/Bienta standard procedures to detect and record abnormalities and macroscopic alterations in internal and external organs. The following organs and parameters were investigated: coat and general external condition of an animal according to the 3-level ranking: good, tolerable and poor; blood color in the vessels: the evaluation was performed immediately after euthanizing the animals. The blood color did not differ between the animals, therefore, this parameter is not mentioned in the results; intestines: presence and amount of gas and feces in intestines; and internal organs (kidneys, thymus, spleen, liver, lungs, heart, uterus and pancreas) weight and condition: color, shape, tissue consistency, morphology peculiarities and mass of the organs were investigated. Statistical evaluation of the toxicity data was performed by a nonparametric statistical analysis (Wilcoxon-Mann-Whitney U test), with the Wilcoxon matched-pairs test for dependent and the Sign test for independent samples. LD $_{50}(50 \%$ lethal dose) values were determined by the probit analysis [43]. The p-value of less than 0.05 was considered statistically significant.

\section{Assessment of phototoxicity of LMB040-closed}

Phototoxicity was analyzed after a single-dose intraperitoneal administration in Balb/c mice keeping the animals under standard dark-light regimen (electrical luminescent light for $12 \mathrm{~h}$, darkness $12 \mathrm{~h}$ ). LMB040-closed was administered at a sub-LD $\mathrm{LD}_{50}$ dose of $55 \mathrm{mg} / \mathrm{kg}$. The animals were kept in semi-darkness during the dosing. After the injection, one group was kept in complete darkness, the other retained a $12 \mathrm{~h}$ light, $12 \mathrm{~h}$ darkness regimen. The complete experimental period was devised as follows: 7 days of acclimatization, 1 day of treatment, 13 additional days of post-treatment observations (21 days in total), the autopsy on the last day. Observations and examination of the animals were done as described for the acute toxicity section above. 


\section{ADME studies}

Pharmacokinetic analysis of LMBO02

C57BL/ 6 mice bearing LLC were used in this study. To establish the allograft, $5 \times 10^{5}$ LLC cells were inoculated into the subcutis of the right leg of each animal 10 days before the study. The tumors at the site of inoculation were palpable by the time of the experiments $(\sim 3-5 \mathrm{~mm}$ in diameter). The animals were randomly assigned to the experimental groups (three animals per time point) before the pharmacokinetic study; all animals fasted for $16 \mathrm{~h}$ before intravenous dosing. LMB002 was administered as a mixture (50:50 mol \%) of the open and closed forms and at a $5 \mathrm{mg} / \mathrm{kg}$ dose, in the volume of $5 \mathrm{ml} / \mathrm{kg}$ of the bodyweight. Co-administration of open and closed photoforms reduced the total number of animals used in the experiment.

Treated animals were injected intravenously with tribromoethanol at the dose of $100 \mathrm{mg} / \mathrm{kg} 2 \mathrm{~min}$ prior to the collection of blood. Blood samples were withdrawn from the retroorbital sinus and were collected in $0.5 \mathrm{ml}$ BD Microtainer blood collection tubes containing $\mathrm{K}_{2}$ salt of ethylenediaminetetraacetic acid as an anticoagulant. Animals were sacrificed by cervical dislocation after the blood samples collection. The blood and tissue specimens (blood plasma, tumor, liver, spleen, lungs, kidneys, heart, colon and brain) were harvested at 15, 30, 60, 120, 240, 480 and 1440 min after administration.

All samples were immediately flash-frozen and stored at $-70^{\circ} \mathrm{C}$ until subsequent analysis. Five hundred microliter of the IS250 solution (Internal Standard, $250 \mathrm{ng} / \mathrm{ml}$ in acetonitrile-methanol mixture 1:1, v/v) was added to the tumor, liver, lungs, kidneys, heart and spleen samples (average tissue weight $100 \mathrm{mg}$ ). The samples were dispersed by a tissue-tearor homogenizer for 30-60 s at $1000 \mathrm{rpm}$. After centrifugation for $4 \mathrm{~min}$ at $14000 \mathrm{rpm}$ on a bench-top centrifuge, $4 \mu \mathrm{l}$ of each supernatant was used for the analysis. Brain samples (average tissue weight $100 \mathrm{mg}$ ) were mixed with $500 \mu \mathrm{l}$ of the IS250 solution $(250 \mathrm{ng} / \mathrm{ml}$ in water-acetonitrile-methanol mixture 2:4:4, v/v/v). These samples were dispersed for $20 \mathrm{~s}$ at $1000 \mathrm{rpm}$. After centrifugation for $3 \mathrm{~min}$ at $14000 \mathrm{rpm}, 4 \mu \mathrm{l}$ of each supernatant was used for the analysis. Liquid plasma samples (average volume $50 \mu \mathrm{l}$ ) were mixed with $200 \mu \mathrm{l}$ of the IS250 solution. Without homogenization, after centrifugation for $4 \mathrm{~min}$ at $6000 \mathrm{rpm}, 1 \mu \mathrm{l}$ of each supernatant was analyzed.

The concentrations of the tested compounds (both photoforms individually) were determined using HPLCMS/MS method. A Shimadzu (Kyoto, Japan) VP HPLC system equipped with isocratic pumps LC-10ADvp, an autosampler SIL-HTc, a sub-controller FCV-14AH and a degasser DGU-14A was used. The mass spectrometric analysis was performed using a Sciex (MA, USA) API 3000 (triple-quadrupole) instrument with electrospray ionization. The data acquisition and system control were performed using Analyst 1.5.2 software from Sciex.

\section{Pharmacokinetic study of LMBO02 with \& without visible light irradiation}

C57BL/6 mice bearing LLC (generated as described above) were used in the experiment. The animals in two groups (21 and 27 mice each) were administered a single-dose LMB002 (IV, $5 \mathrm{mg} / \mathrm{kg}$ in Cremophor EL - Saline [20\%:80\% v/v]), then the smaller group was kept in darkness as a control. The animals of the larger group received irradiation at the tumor site $(660 \mathrm{~nm}$, a semiconductor laser light source LIKA-Surgeon, Photonica Plus, Ukraine; $15 \mathrm{~min}$, irradiance $100 \mathrm{~mW} / \mathrm{cm}^{2}, 3 \mathrm{~h} 45 \mathrm{~min}$ after the dosing). The blood plasma and tumor tissue were harvested at $0.5,2,4.5,6,8,12,24,36$ and $48 \mathrm{~h}$ (three animals per each time point). All samples were prepared and analyzed, as described in the section above.

\section{Analysis of stability in mouse plasma for LMB002 \& LMB033}

Nonsterile mouse plasma with heparin (MPH07-0500 from Equitech-Bio, TX, USA) was used. Individual photoforms of the tested and reference compounds (verapamil and propantheline bromide) were analyzed at five time points over 120 min using HPLC-MS/MS for detection (see above). Both positive- and negative-ion modes of a TurbolonSpray (Sciex) ionization source were used.

Co-incubations with mouse plasma were carried out in five aliquots of $70 \mu \mathrm{l}$ each (one for each time point) in duplicates. Tested compounds $(1 \mu \mathrm{M}$, final DMSO concentration of $1 \%)$ were incubated at $37^{\circ} \mathrm{C}$ with shaking at $100 \mathrm{rpm}$. The reactions were stopped by adding $420 \mu \mathrm{l}$ of $90 \%$ acetonitrile-water with subsequent sedimentation of plasma proteins at $5500 \mathrm{rpm}$ for $5 \mathrm{~min}$. Obtained supernatants were analyzed: a percentage of tested compounds remaining after the incubation were determined and their half-lives $\left(\mathrm{t}_{1 / 2}\right)$ were calculated. 


\section{Assessment of metabolic stability in mouse liver microsomes for LMB002 \& LMB033}

Metabolic stability of individual photoforms of the tested and reference compounds (imipramine and propranolol) was determined upon co-incubation with mouse liver microsomes at five time points over 40 min using HPLCMS/MS for detection (see above).

Microsomes were isolated from pooled perfused livers of Balb/c male mice according to the standard protocol [44]. Each batch of microsomes was first tested for quality using imipramine, propranolol and verapamil as references. Co-incubation with microsomes was carried out in 96-well plates in five aliquots of $40 \mu \mathrm{l}$ per compound (one for each time point) in duplicates. Incubation medium contained phosphate-buffered saline (PBS), $100 \mathrm{mM}, \mathrm{pH}$ 7.4, $\mathrm{MgCl}_{2}(3.3 \mathrm{mM})$, NADPH (3 mM), glucose-6-phosphate $(5.3 \mathrm{mM})$ and glucose-6-phosphate dehydrogenase $(0.67$ units $/ \mathrm{ml})$ with $0.42 \mathrm{mg}$ of liver microsomal protein per $\mathrm{ml}$. Control wells were supplemented with PBS instead of the NADPH-cofactor system. Tested compounds $(2 \mu \mathrm{M}$, final solvent concentration $1.6 \%)$ were coincubated with microsomes at $37^{\circ} \mathrm{C}$, shaking at $100 \mathrm{rpm}$. The reactions were stopped by adding 12 volumes of $90 \%$ acetonitrile-water, followed by protein sedimentation $(5500 \mathrm{rpm}$ for $3 \mathrm{~min})$. Supernatants were analyzed: the elimination constants $\left(\mathrm{k}_{\mathrm{el}}\right)$, half-lives $\left(\mathrm{t}_{1 / 2}\right)$ and intrinsic clearance values $\left(\mathrm{Cl}_{\text {int }}\right)$ were calculated from plots of $\ln ($ AUC) versus time, using linear regression analysis.

\section{Mouse plasma protein binding assay for LMB002 \& LMB033}

Binding to mouse plasma proteins of individual photoforms of photoswitchable compounds along with the reference compound (verapamil) was assessed using HPLC-MS/MS (see above). The experiment was performed by spiking tested compounds at a concentration of $1 \mu \mathrm{M}$ into the intact mouse plasma and dialyzing against PBS. Concentrations of the compounds in plasma and PBS were determined to calculate the plasma protein-bound fraction. The assay was performed in a 96-well dialysis unit (HTD96b dialyzer). Each well consisted of two chambers separated by a vertically aligned dialysis membrane (MWCO 12-14 kDa). Nondiluted plasma (120 $\mu \mathrm{l})$ spiked with the tested compound $(1 \mu \mathrm{M}$, final DMSO concentration $1 \%)$ was added to one chamber and the same volume of PBS buffer ( $\mathrm{pH}$ 7.4) to the other chamber. The dialysis unit was covered with an adhesive sealing film and incubated at $37^{\circ} \mathrm{C}$ with shaking at $100 \mathrm{rpm}$ for $5 \mathrm{~h}$. An aliquot from each chamber had been mixed with the same volume of the blank opposite matrix. Before HPLC-MS/MS analysis, the samples were diluted tenfold with acetonitrile with subsequent plasma protein sedimentation at $6000 \mathrm{rpm}$ for $5 \mathrm{~min}$ and the supernatants were analyzed.

\section{CYP450 inhibition study for LMB002 \& LMB033}

Assessment of the inhibitory activity was done on a major human CYP450 enzyme panel (1A2, 2C9, 2C19, 2D6 and $3 \mathrm{~A} 4)$ at a single tested compound concentration of $10 \mu \mathrm{M}$. The experiment was performed using fluorogenic substrates corresponding to CYP450 enzymes (Vivid ${ }^{\circledR}$ CYP450 Screening Kits, Thermo Fisher Scientific, MA, USA) with minor changes to the manufacturer's protocols. In brief, the tested compounds were first dissolved in DMSO at $1 \mathrm{mM}$ and diluted in Vivid Reaction Buffer I to $2.5 \times$ concentration $(25 \mu \mathrm{M})$. These solutions were mixed with the Vivid Master Pre-mix Reagent (a CYP450, an oxidoreductase, and an NADP+ regeneration system consisting of glucose-6-phosphate and glucose-6-phosphate dehydrogenase). After 10 min pre-incubation, the reaction was initiated by the addition of a mixture of NADPH and an appropriate Vivid CYP450 substrate. The plate was incubated for the desired reaction time (25 min for CYP1A2, CYP2C9, CYP2D6, CYP3A4 and 60 min for CYP2C19) after which the Vivid Stop Reagent (0.5 M Tris base) was added and fluorescence was recorded with a SpectraMax ${ }^{\circledR}$ Paradigm ${ }^{\circledR}$ Multi-Mode Microplate Reader (Molecular Devices, CA, USA). All experiments were performed in quadruplicates.

\section{Inhibition of hERG}

All experiments were performed using Predictor ${ }^{\circledR} h E R G$ fluorescence Polarization Assay from Invitrogen (Thermo Fisher Scientific, MA, USA), following the manufacturer's instructions. 'Sigmoidal dose-response (variable slope)' function of the GraphPad Prism software was used for the calculation of $\mathrm{IC}_{50}$. The $\mathrm{IC}_{50}$ value for E-4031 (assay reference compound) was found to be approximately $25 \mathrm{nM}$, in accordance with the literature. Three dilutions of the tested compounds were studied: 1,5 and $25 \mu \mathrm{M}$. For the photoisomerizable compounds, both photoforms were assessed separately. The set of positive (no tracer added, $0 \%$ tracer displacement, maximum assay polarization value) and negative controls $(30 \mu \mathrm{M}$ of E-4031; 100\% tracer displacement, minimum assay polarization value) were used to calibrate the assay. All measurements were performed in quadruplicates. 


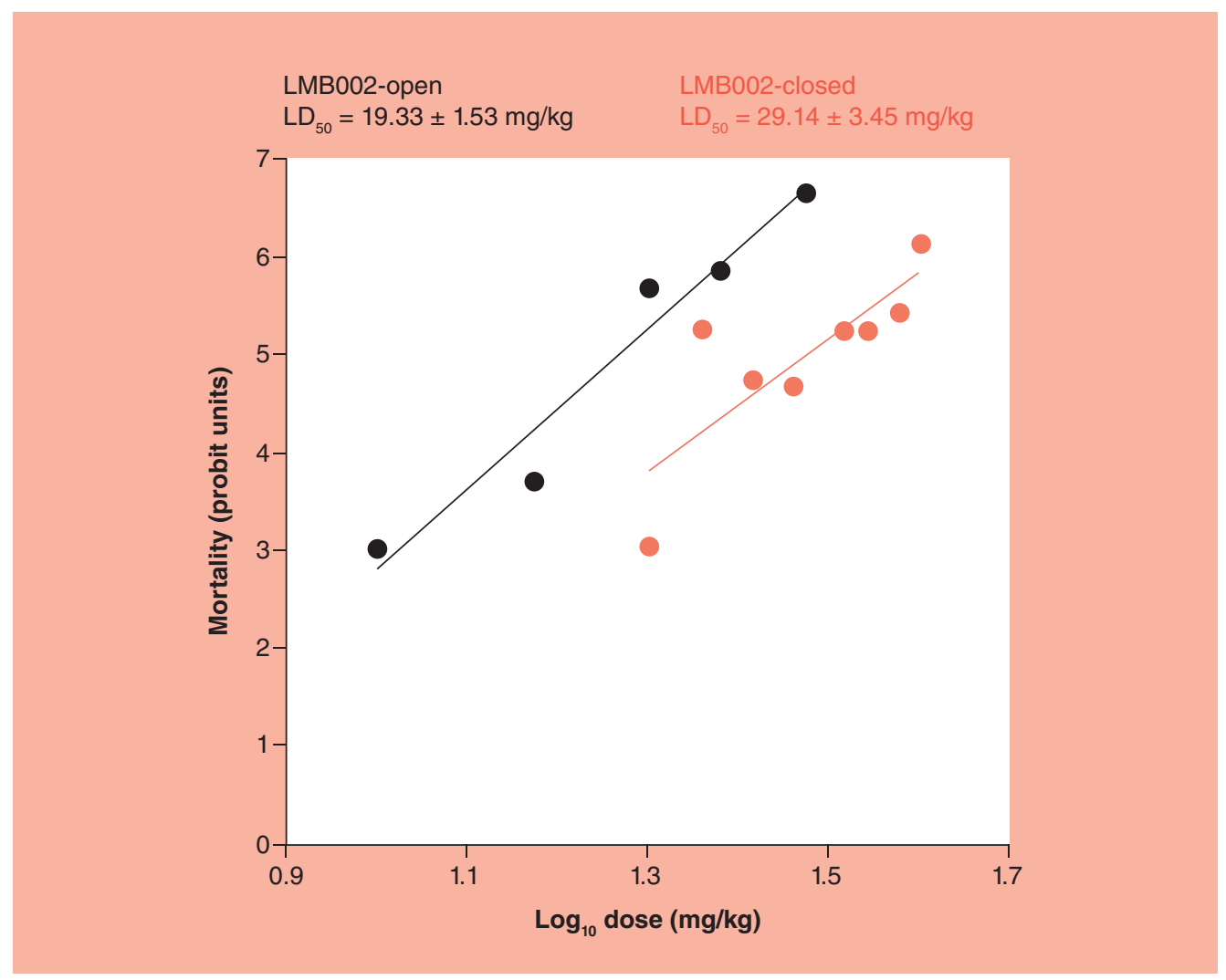

Figure 2. Regression lines of dose-probit mortality data and the calculated LD $_{50}$ values for the two photoforms of LMB002 under investigation. This was determined in female C57BL/6 mice after single-dose intravenous administration.

\section{Results \& discussion}

The open form of compound LMB002 was reported to have high in vitro cytotoxicity (low $\mathrm{IC}_{50}$ values) against several human cancer cell lines that were similar to that of the prototype molecule, GS [13]. The closed form of this compound demonstrated up to eight-times lower cytotoxicity against the same cell lines. The toxicity against human erythrocytes $\left(50 \%\right.$ hemolysis, $\mathrm{HC}_{50}$ ) was also lower for the closed photoform of LMB002. The ratio of $\mathrm{HC}_{50}$ for the closed isomer to the $\mathrm{IC}_{50}$ value for the open isomer (defined as the 'phototherapeutic index' in [13]) was generally within the range of $8-11$, depending on the cancer cell line being investigated.

Hemolysis is generally postulated to be the prime mechanism of toxicity for GS and its analogs in vivo. Therefore, the phototherapeutic index defined above seemed to be a useful indicator for assessing the in vivo safety data for this class of compounds. To verify this assumption, the $\mathrm{LD}_{50}$ values for $\mathrm{LMB} 002$ in $\mathrm{C} 57 \mathrm{BL} / 6$ mice were determined for both the open and closed forms and the results were compared with the $\mathrm{LD}_{50}$ values obtained for the prototype molecule, GS.

Although $\mathrm{LD}_{50}$ values are rarely determined during the preclinical studies of drug candidates nowadays, its use in this study seemed justified since these values were very reliable indication of acute toxicity associated with the tested compounds. We found that the $\mathrm{LD}_{50}$ values of the two photoforms of $\mathrm{LMB} 002$ were indeed different: $19.3 \pm 1.5 \mathrm{mg} / \mathrm{kg}$ for the open form and $29.1 \pm 3.5 \mathrm{mg} / \mathrm{kg}$ for the closed form. The toxicity of the open form was almost the same as that of the prototype GS $(18.4 \pm 1.1 \mathrm{mg} / \mathrm{kg})$, but the closed form was notably less toxic. This result proved that LMB002 could, in principle, be used in photopharmacological applications if administered as the less-toxic (closed) isomer and subsequently converted to the active (open) form in vivo. However, the observed differences between the $\mathrm{LD}_{50}$ values for both $\mathrm{LMB} 002$ photoforms $(\approx 1.5$-fold), at least when the compound was administered intravenously, were much smaller than the cytotoxicity difference observed in vitro (up to twentyfold). The less cytotoxic closed form, thus, turned out to be unexpectedly toxic in vivo, so that there was a wide range of concentrations at which both photoforms induced a mortality rate of more than 30\% (Figure 2). Although LMB002 


\begin{tabular}{|c|c|c|c|c|c|c|c|c|c|}
\hline Tissue & $T_{\max }, \min$ & $C_{\max }, \mathrm{ng} / \mathrm{ml}$ & $\begin{array}{l}\mathrm{AUC} C_{0 \rightarrow \mathrm{t}}=480 \\
\mu \mathrm{g} \cdot \mathrm{min} / \mathrm{ml}\end{array}$ & $\begin{array}{l}\mathrm{AUC}_{0 \rightarrow \infty} \\
\mu \mathrm{g} \cdot \mathrm{min} / \mathrm{ml}\end{array}$ & $T_{1 / 2}, \min$ & $\mathrm{K}_{\mathrm{el}}, \min ^{-1}$ & MRT, min & $\mathrm{V}_{\mathrm{d}} \mathrm{mL} / \mathrm{kg}$ & $\mathrm{CL} \mathrm{ml} / \mathrm{min} / \mathrm{kg}$ \\
\hline \multicolumn{10}{|c|}{ LMB002-closed } \\
\hline Plasma & 30 & 19200 & 3120 & 3430 & 154 & 0.0045 & 132 & 324 & 1.46 \\
\hline Liver & 60 & 6010 & 1730 & 1820 & 340 & 0.00204 & 302 & $\mathrm{~N} / \mathrm{A}$ & $\mathrm{N} / \mathrm{A}$ \\
\hline Kidney & 60 & 19800 & 6900 & 7060 & 265 & 0.00261 & 291 & $N / A$ & $N / A$ \\
\hline Heart & 30 & 5690 & 924 & 978 & 115 & 0.00605 & 143 & $\mathrm{~N} / \mathrm{A}$ & $\mathrm{N} / \mathrm{A}$ \\
\hline Spleen & 30 & 3410 & 524 & 568 & 135 & 0.00512 & 152 & $\mathrm{~N} / \mathrm{A}$ & $\mathrm{N} / \mathrm{A}$ \\
\hline Tumor & 240 & 2900 & 2860 & 10100 & 2630 & 0.00263 & 716 & $\mathrm{~N} / \mathrm{A}$ & $\mathrm{N} / \mathrm{A}$ \\
\hline Lungs & 30 & 1210 & 249 & 327 & 524 & 0.00132 & 467 & N/A & N/A \\
\hline Kidney & 30 & 796 & 618 & 980 & 979 & 0.000708 & 564 & $N / A$ & N/A \\
\hline Heart & 30 & 697 & 274 & 648 & 1920 & 0.00036 & 576 & $N / A$ & N/A \\
\hline Spleen & 60 & 580 & 420 & 959 & 1640 & 0.000423 & 635 & $\mathrm{~N} / \mathrm{A}$ & $\mathrm{N} / \mathrm{A}$ \\
\hline Tumor & 30 & 190 & 82.7 & 99.3 & 501 & 0.00138 & 438 & $N / A$ & $N / A$ \\
\hline
\end{tabular}

was shown to exert a photopharmacological antitumor efficacy in vivo in the dose lower than the $\mathrm{LD}_{50}$ measured here $(9.1 \mathrm{mg} / \mathrm{kg}$ [12]), there was still much to be desired in terms of the safety parameters of this compound. Therefore, we concluded that in the mouse model, the advantage gained in the in vivo safety parameters due to photocontrol of LMB002 turned out to be quite small.

One of the reasons for the small difference in the $\mathrm{LD}_{50}$ values for the photoisomers of LMB002 could be their unfavorable pharmacokinetic parameters. To confirm this, we first determined the time-dependent distribution of the two LMB002 photoforms in the blood plasma and tissues of the C57BL/6 mice at a sub-lethal intravenous dose. The results are summarized in Table 1.

As can be observed from the results in Table 1 , the maximum concentration $\left(\mathrm{C}_{\max }\right)$ of the LMB002-closed form in plasma and kidney tissues was the highest among all the organs measured. High plasma concentrations are of particular concern since they rose to a dangerous level $(19.2 \mu \mathrm{g} / \mathrm{ml})$ at which significant hemolysis could be induced [13]. Notably, high concentrations of LMB002-closed form persisted in the blood, liver and kidney for quite a long time with half-lives $\left(\mathrm{T}_{1 / 2}\right)$ of 154,340 and $265 \mathrm{~min}$, respectively; this could explain the relatively low $\mathrm{LD}_{50}$ values associated with this compound.

The slow clearance rate of LMB002 could, in part, be explained by its interaction with plasma proteins. To evaluate this, we measured mouse plasma protein binding for a reference compound, verapamil and for both photoforms of LMB002. Indeed, we found that irrespective of the photoisomeric state, more than $99 \%$ of LMB002 was bound when compared with $84 \%$ for verapamil (see Supplementary Material).

An additional factor that could be used to explain the peculiar pharmacokinetic behavior of LMB002 could be its in vivo stability leading to low elimination constants. This assumption was verified by measuring the mouse blood plasma stability and the microsomal stability of LMB002. As was anticipated, given the cyclic structure of the peptide, both experiments confirmed that there was high metabolic stability of the compound under physiological conditions (see Supplementary Material). A low-intensity signal for the $[M+16]$ metabolite was detected upon prolonged co-incubation of the LMB002-closed form with the microsomes. It was theorized that this signal corresponded to an oxidized peptide formed during the incubation process. However, its intensity was very low; there were negligible changes in the overall concentration of the intact LMB002-closed form even after 40 min of incubation. 


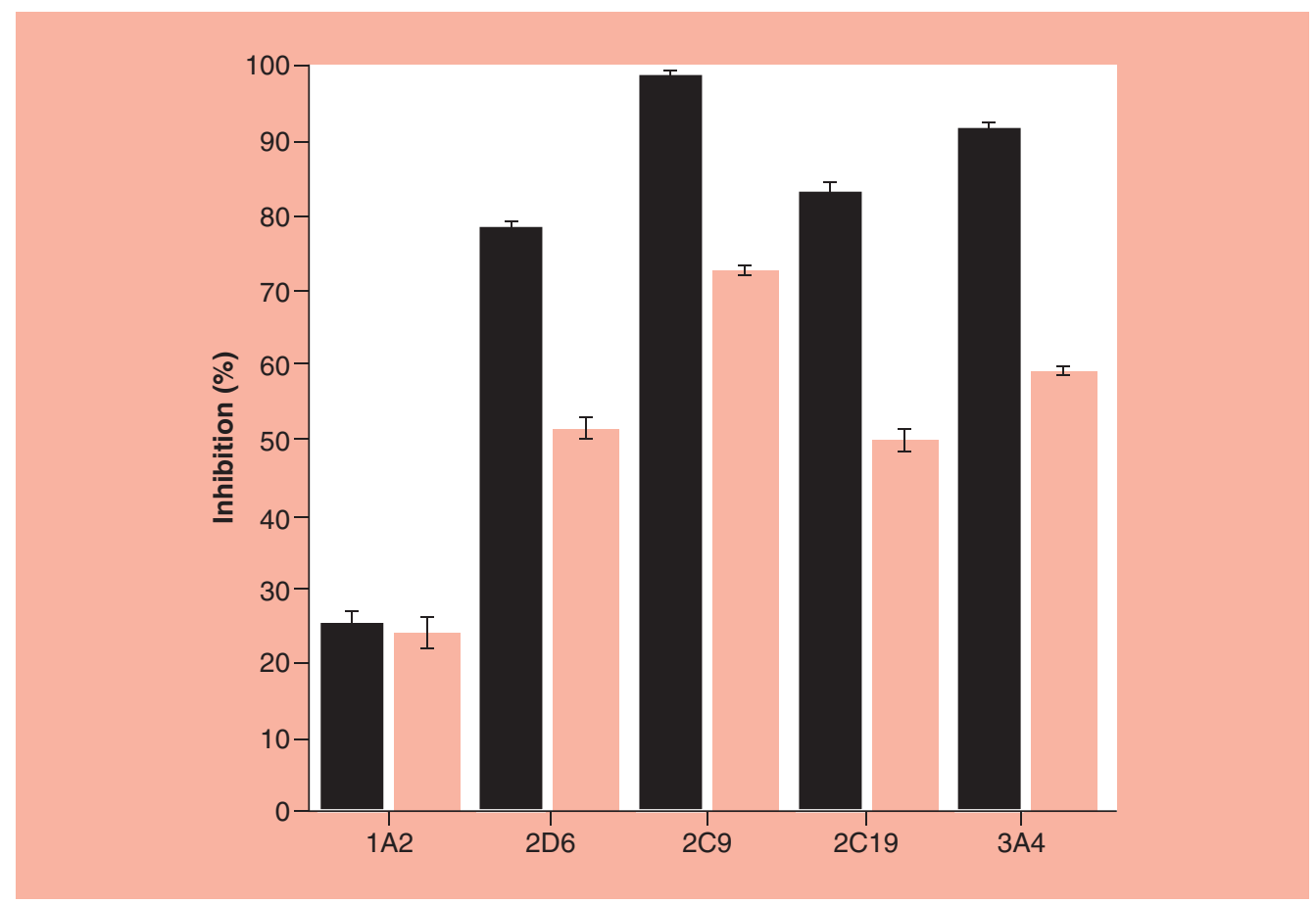

Figure 3. CYP450 inhibition profile for LMB002. The data points are collected at a single $10 \mu \mathrm{M}$ concentration. Values for LMB002-open form are presented as black bars, whereas the values for the LMB002-closed form are presented as orange bars.

The extent of inhibition of CYP450 enzymes by LMB002 was also examined. Both photoforms, LMB002open and LMB002-closed, significantly inhibited all tested CYP isozymes (1A2, 2C9, 2C19, 2D6 and 3A4) at a concentration of $10 \mu \mathrm{M}$ (Figure 3).

Another potential pharmacokinetic problem, often pointed out by critics of photopharmacology, is the distribution of the active and toxic photoisomer across the patient's entire body via the bloodstream after photoactivation. It was theorized that this might have further contributed to the overall high toxicity of the treatment, thereby diminishing the inherent photopharmacological benefits. We found that this problem was overestimated, at least in the case of the cytotoxic amphiphilic peptidomimetics derived from GS. The concentration of both photoisomers in plasma and tumor tissues was measured after an intravenous administration of the LMB002 closed form to mice bearing engrafted LLC; this was done before and after application of the irradiation treatment of the primary tumors. A control experiment without irradiation was also conducted. The results in Figure 4 clearly reveal that the concentration of the open form in the tumors sharply increased after the irradiation treatment, but remained negligible in the blood plasma.

Apart from the biodistribution-related issues, another cause for additional toxicity associated with the closed form of LMB002 could be the occurrence of strong nonspecific interactions with targets known to cause severe side effects. One such interaction that should routinely be checked in preclinical studies for new chemotherapeutic agents is with the hERG protein. This is a potassium channel that serves one of the central regulators of cardiac rhythm and its inhibition was found to have severe or even fatal side effects due to the induction of an irregular heartbeat [45]. Using a fluorescence polarization assay, we measured the binding affinity of the LMB002-closed form to hERG at three different concentrations and compared these data with those of the prototype peptide. The results (Supplementary Table 54) suggested that the hERG inhibition could become dangerously high, even exceeding the levels of the reference compounds and GS. Consequently, hERG inhibition-related cardiotoxicity might contribute to the overall toxicity of our compound in vivo. Even though this suggestion should be verified with more relevant functional assays for this target, it is still necessary to minimize incidences of this unspecific protein binding by modifying the LMB002 molecule accordingly.

In summary, the nonoptimal pharmacokinetic properties of the LMB002-closed form, especially the rapid increase of its concentration in plasma, liver and kidney to dangerous levels after administration, can be used to 


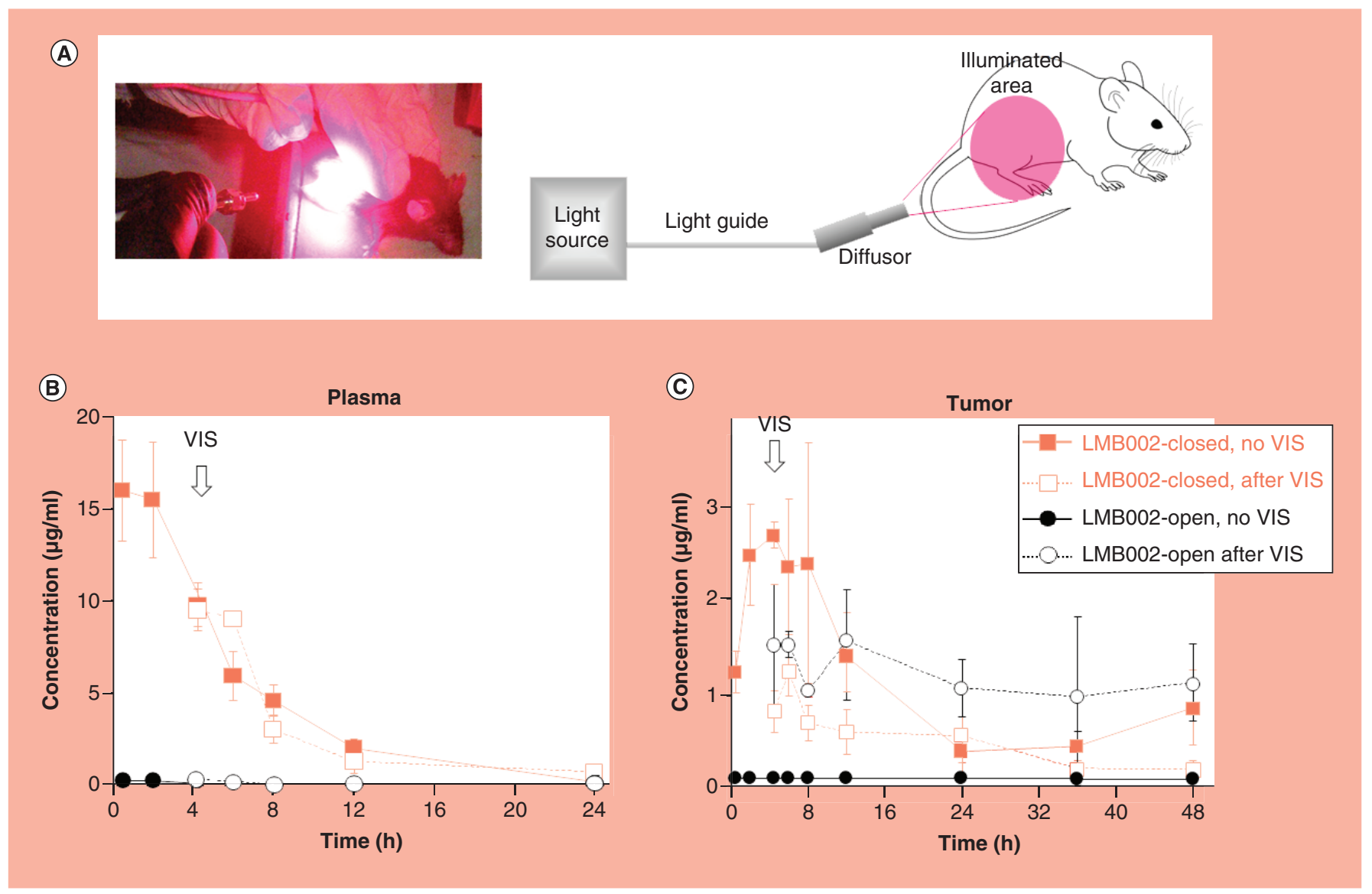

Figure 4. Photoactivation of LMB002 in vivo. (A) The experimental setup; the concentration of LMB002-closed and LMB002-open forms in plasma (B) and tumor (C) before and after irradiation (shown as marked VIS and hollow arrows in the figure, $\lambda_{\max } 660 \mathrm{~nm}, 15 \mathrm{~min}$, $100 \mathrm{~mW} / \mathrm{cm}^{2}$ ). Data for the initially applied LMB002-closed form are highlighted in color.

VIS: Visible light.

explain its unexpectedly high in vivo toxicity values. This finding prompted us to further modify the compound in order to improve its safety profile.

Applying the design principles established in [13], we prepared several novel GS analogs, namely LMB033, LMB037, LMB039 and LMB040. Like LMB002, these compounds also demonstrated high cytotoxicity against a variety of human cancer cell lines in their open forms (Table 2). For most of the cell lines, these novel compounds demonstrated more significant differences in the cytotoxic activities of the closed versus the open forms. For one of the compounds, $\mathrm{LMB} 033$, the ratio $\mathrm{IC}_{50}$ (closed)/ $\mathrm{IC}_{50}$ (open) was up to three-times higher than that observed for LMB002, independent of the cell line used; ultimately, this ratio peaked at 60. The activity of LMB033-open was as high as that of the GS prototype against all the cell lines investigated, including the doxorubicin-resistant human lung adenocarcinoma cell line MOR/0.2R.

The hemolytic activity of both photoforms for compounds LMB033 and LMB040 was markedly lower than that of LMB002 and the prototype (see Supplementary Material). Hence, their in vitro phototherapeutic index could be improved simply by chemical modifications. We were eager to see whether this would also result in more notable differences in the in vivo toxicity for the novel compounds. Both Table 3 and Figure 5 present the in vivo $\mathrm{LD}_{50}$ values measured for the novel analogs in female C57BL/6 mice upon intravenous administration. We were pleased to see that the in vivo toxicity could, indeed, be decreased by structural modifications, as the difference in the toxicity values between the open and closed forms was increased. This gap was particularly pronounced for compounds LMB037 and LMB040. The dose-dependent mortality curves are sufficiently separate (Figure 5A \& B when compared with Figure 2 for LMB002), so that the dosages for therapy could be safely spread over a wide region. Notably, the toxicity profile depended significantly on the means of administration, confirming the theory 


\begin{tabular}{|c|c|c|c|c|c|c|c|c|c|c|c|}
\hline Compound & HeLa & Hep G2 & COLO 205 & MCF-7 & MOR & $\mathrm{MOR} / 0.2 \mathrm{R}$ & H69 & WM 1158 & HEK 293 & $\mathrm{CHO}$ & MIA-PaCa-2 \\
\hline LMB002-open & 6 & 7 & 6 & 6 & 4 & 5 & 6 & 6 & 12 & 12 & 6 \\
\hline LMB002-closed & 65 & 96 & 128 & 64 & 64 & 80 & 64 & 100 & 192 & 192 & 50 \\
\hline LMB002-closed/open ratio & 10.8 & 13.7 & 21.3 & 10.7 & 16 & 16 & 10.7 & 16.7 & 16 & 16 & 8.3 \\
\hline LMB033-open & 4.2 & ND & 5 & 6 & 5.5 & 3 & 4 & 4 & ND & ND & 5 \\
\hline LMB033-closed & 110 & ND & 300 & 128 & 96 & 64 & 96 & 200 & ND & ND & 160 \\
\hline LMB033-closed/open ratio & 26.2 & ND & 60 & 21.3 & 17.5 & 21.3 & 24 & 50 & ND & ND & 32 \\
\hline LMB03-open & 2 & ND & ND & ND & ND & ND & ND & ND & ND & ND & ND \\
\hline LMB037-closed & 40 & ND & ND & ND & ND & ND & ND & ND & ND & ND & ND \\
\hline LMB037-closed/open ratio & 20 & ND & ND & ND & ND & ND & ND & ND & ND & ND & ND \\
\hline LMB039-open & 5 & ND & ND & ND & ND & ND & ND & ND & ND & ND & ND \\
\hline LMB039-closed & 96 & ND & ND & ND & ND & ND & ND & ND & ND & ND & ND \\
\hline LMB039-closed/open ratio & 19.2 & ND & ND & ND & ND & ND & ND & ND & ND & ND & ND \\
\hline LMB040-open & 16 & 8 & ND & 22 & 6 & ND & ND & ND & 24 & 24 & 8 \\
\hline LMB040-closed & 200 & 96 & ND & 192 & 64 & ND & ND & ND & 192 & 192 & 256 \\
\hline LMB040-closed/open ratio & 12.5 & 12 & ND & 8.7 & 10.7 & ND & ND & ND & 8 & 8 & 32 \\
\hline
\end{tabular}

\begin{tabular}{|c|c|c|c|c|c|}
\hline & LMB002 & LMB033 & LMB037 & LMB039 & LMB040 \\
\hline $\mathrm{LD}_{50}$ (open form) & $19.3 \pm 1.5$ & $28.6 \pm 1.4$ & $22.4 \pm 2.5$ & $23.9 \pm 2.3$ & $20.2 \pm 1.5$ \\
\hline $\mathrm{LD}_{50}$ (closed form) & $29.1 \pm 3.5$ & $48.0 \pm 2.0$ & $39.8 \pm 5.3$ & $36.4 \pm 3.7$ & $44.0 \pm 7.2$ \\
\hline Closed/open ratio & 1.5 & 1.7 & 1.8 & 1.5 & 2.2 \\
\hline
\end{tabular}

that an unfavorable pharmacokinetic profile was the leading cause of the high in vivo toxicity of our compounds. Accordingly, LMB040 that had been administered intraperitoneally had higher $\mathrm{LD}_{50}$ values (Figure $5 \mathrm{C}$ ). This administration route was presumably much safer due to slower absorption of the compound than that observed in the case of the bolus intravenous injection.

Moreover, one of the analogs, LMB040-closed form, demonstrated significantly decreased tracer displacement in the hERG-binding fluorescent assay (see Supplementary Material), indicating that this feature had also been improved by structural modifications. This could presumably be optimized further in future studies.

Finally, we decided to address the issue of phototoxicity as it is another safety concern plaguing possible photopharmacological drug candidates, particularly in light of the fact that there is no regulatory consensus for this class of compounds so far. Long-term phototoxicity is known to be a severe problem in photodynamic therapy, a similar treatment modality which is already in clinical use [46-48]. Similar to photopharmacology, photodynamic therapy is based on the photoactivation of an endogenous treatment agent, such as molecular oxygen, in tumors or infected tissues. When systemically applied, the photosynthesizers which mediate this photoactivation tended to accumulate in the external parts of the patients' bodies (e.g., in the skin and other tissues/organs) exposed to light. In these cases, background irradiation caused severe skin and organ damage [47].

The phototoxicity of the LMB0 40 was tested on Balb/c mice. The compound was administered intraperitoneally at a high dose close to its $\mathrm{LD}_{50}$ value $(55 \mathrm{mg} / \mathrm{kg})$ and the resulting toxicity data were compared between for the animals kept in darkness versus those kept under a normal daylight regimen. During the first $2 \mathrm{~h}$ of observation, half of the animals in the 'dark' group exhibited severe signs of toxicity (piloerection, respiratory failure, closed or sunken eyes, inactivity). Since these signs were acute, no further observation was noted during any succeeding monitoring sessions. The animals in the 'daylight' group exhibited the same signs of toxicity for at least $6 \mathrm{~h}$ or even longer. These toxicity indications gradually faded and even disappeared altogether near the last observation period for all test subjects. Hence, the phototoxicity of photopharmacology drugs, at least of the type of compounds 

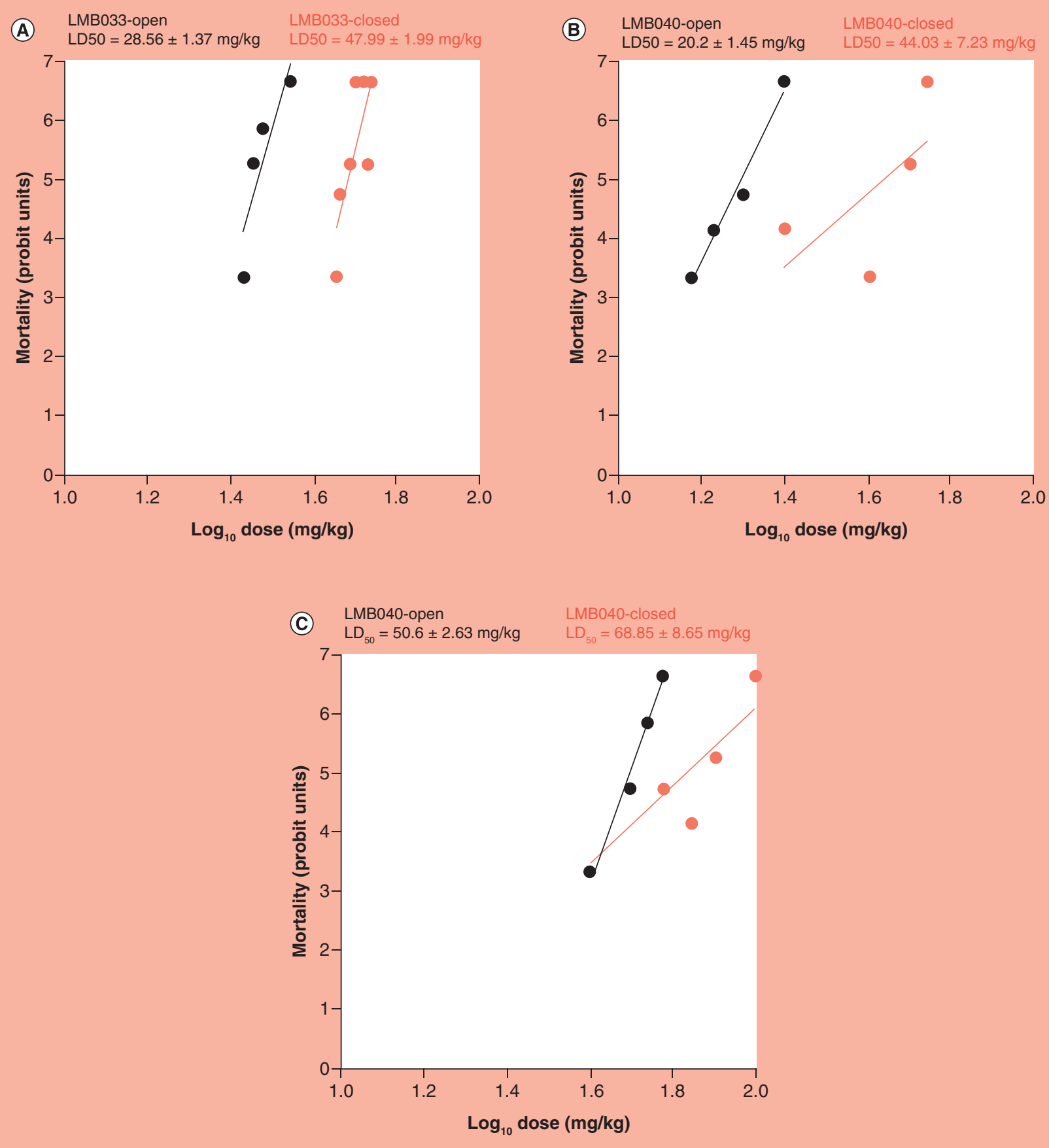

Figure 5. In vivo toxicity of novel gramicidin S analogs. Rodent dose-mortality data for both photoforms of (A) LMB033 (intravenous administration), (B) LMB040 (intravenous administration), and (C) LMB040 (intraperitoneal administration). The closed isomers are highlighted with color.

studied here, should certainly be taken into account during the drug development process. Any such therapy course in the future should ensure that patients are protected from direct daylight exposure for extended periods of time.

\section{Future perspective}

Implementing principles of photopharmacology will open an extra avenue in terms of drug safety in the treatment of human diseases. This addendum justifies further efforts in the development of photocontrollable drugs and the 
potentially high costs of their application due to the need for specialized light sources and trained clinical personnel. It is our belief that these efforts will be rewarding in the long term, eventually resulting in the first photocontrollable drugs for human use. This category of therapeutics will find an important place in the future drug market.

\section{Summary points}

- The in vivo safety of photocontrollable compounds was, indeed, higher than that of nonphotocontrollable prototypes, that we have demonstrated here using one particular representative.

- In vitro experiments failed to adequately predict the safety margins of a drug target due to the crucial role of the therapeutic agent's pharmacokinetic profile in in vivo toxicity tests. In each particular case, the safety of the photopharmacology drug candidate should be comprehensively checked in preclinical tests for animals in order to evaluate all photoisomeric forms and compare them with the nonphotocontrollable analogs where possible.

- Structural modifications can be used to improve the phototherapeutic index of a photocontrollable compound and its in vivo safety profile.

- The mode of administration of a photocontrollable compound significantly influences its toxicity profile.

- Photocontrollable compounds can efficiently be activated locally at the sites of lesions (e.g., in tumors) by a tissue-benign red light. This activation does not necessarily lead to fast redistribution of the activated compound throughout the whole body. Thus, the activated compound can be gradually cleared from the treated organism.

- The phototoxicity of a photocontrollable compound should always be taken into account and checked during photopharmacological drug development processes.

Supplementary data

To view the supplementary data that accompany this paper please visit the journal website at: www.futurescience.com/doi/suppl/10.4155/fdd-2019-0033

Author contributions

O Babii, S Afonin, AS Ulrich and IV Komarov performed conceptualization; O Babii, S Afonin, LV Garmanchuk, V Yurchenko, S Zozulya, I Pishel and IV Komarov performed methodology; O Babii, S Afonin, T Schober, LV Garmanchuk, LI Ostapchenko, V Yurchenko, S Zozulya, I Pishel, AS Ulrich and IV Komarov performed formal analysis; O Babii, S Afonin, T Schober, LV Garmanchuk, V Yurchenko, O Tarasov, I Pishel and IV Komarov performed investigation; IV Komarov prepared the original draft; O Babii, S Afonin, AS Ulrich and IV Komarov did review and editing; O Babii, S Afonin, LI Ostapchenko, S Zozulya, AS Ulrich and IV Komarov performed supervision.

\section{Acknowledgments}

We thank P Wadhwani (KIT, Karlsruhe) for his help and assistance via the peptide synthesis facility. The authors would like to thank Enago (www.enago.com) for the English language review.

\section{Financial \& competing interests disclosure}

The authors acknowledge EU funding by the EU H2020-MSCA-RISE-2015 through the PELICO project (grant 690973). This work was supported by the DFG-GRK 2039 (S Afonin, T Schober and AS Ulrich) and by the BMBF VIP+ (O Babii and AS Ulrich). O Babii, S Afonin, AS Ulrich and IV Komarov are inventors on the issued patent family: "Peptidomimetics possessing photocontrolled biological activity" (WO2014127919 [A1], EP2958934 [B1], US9481712 [B2], UA113685 [C2]). O Babii, S Afonin, T Schober and IV Komarov are founders and shareholders of Lumobiotics $\mathrm{GmbH}$. The authors have no other relevant affiliations or financial involvement with any organization or entity with a financial interest in or financial conflict with the subject matter or materials discussed in the manuscript apart from those disclosed.

No writing assistance was utilized in the production of this manuscript.

Information pertaining to writing assistance

The authors would like to thank Enago (www.enago.com) for the English language review.

Ethical conduct of research

Animal care and experimental procedures were performed following the 8th edition (2011) of the 'Guide for the Care and Use of Laboratory Animals' by the National Research Council (USA) and under Article 6 of the Law of Ukraine 'On Medicines'. This study has been approved by the Bioethics Commission of Educational and Scientific Centre 'Institute of Biology and Medicine' of 
the Taras Shevchenko National University of Kyiv. Minimum possible numbers of animals have been used in all studies a nd the literature data were used wherever available.

\section{Open access}

This work is licensed under the Attribution-NonCommercial-NoDerivatives 4.0 Unported License. To view a copy of this license, visit http://creativecommons.org/licenses/by-nc-nd/4.0/

\section{References}

Papers of special note have been highlighted as: $\bullet$ of interest; $\bullet \bullet$ of considerable interest

1. Velema WA, Szymański W, Feringa BL. Photopharmacology: beyond proof of principle. J. Am. Chem. Soc. 136(6), 2178-2191 (2014).

-• A seminal review on photopharmacology.

2. Broichhagen J, Frank JA, Trauner D. A roadmap to success in photopharmacology. Acc. Chem. Res. 48(7), 1947-1960 (2015).

3. Lerch MM, Hansen MJ, van Dam GM, Szymański W, Feringa BL. Emerging targets in photopharmacology. Angew. Chem. Int. Ed. 55(37), 10978-10999 (2016).

- A review defining the possible treatment strategies in photopharmacology.

4. Hüll K, Morstein J, Trauner D. In vivo photopharmacology. Chem. Rev. 118(21), 10710-10747 (2018).

5. Casparro SP, Chan G, Edelson RL. Phototherapy and photopharmacology. Yale J. Biol. Med. 58(6), 519-534 (1985).

6. Tochitsky I, Helft Z, Meseguer V et al. How azobenzene photoswitches restore visual responses to the blind retina. Neuron 92(1), $100-113$ (2016).

7. Tochitsky I, Trautman J, Gallerani N, Malis JG, Kramer RH. Restoring visual function to the blind retina with a potent, safe and long-lasting photoswitch. Sci. Rep. 7, 45487 2017).

8. Szymański W. Beyond photodynamic therapy: light-activated cancer chemotherapy. Curr. Med. Chem. 24(42), 4905-4950 (2017).

9. Velema WA, Van Der Berg JP, Hansen MJ, Szymański W, Driessen AJ, Feringa BL. Optical control of antibacterial activity. Nat. Commun. 5(11), 924-928 (2013).

10. Brieke C, Rohrbach F, Gottschalk A, Mayer G, Heckel A. Light-controlled tools. Angew. Chem. Int. Ed. 51(34), 8446-8476 (2012).

11. Szymański W, Beierle JM, Kistemaker HA, Velema WA, Feringa BL. Reversible photocontrol of biological systems by the incorporation of molecular photoswitches. Chem. Rev. 113(8), 6114-6178 (2013).

12. Babii O, Afonin S, Garmanchuk LV et al. Direct photocontrol of peptidomimetics: an alternative to oxygen-dependent photodynamic cancer therapy. Angew. Chem. Int. Ed. 55(18), 5493-5496 (2016).

13. Babii O, Afonin S, Ishchenko AY et al. Structure-activity relationships of photoswitchable diarylethene-based $\beta$-hairpin peptides as membranolytic antimicrobial and anticancer agents. J. Med. Chem. 61(23), 10793-10813 (2018).

-. In vitro and in vivo demonstration of photopharmacology efficacy on rodents.

14. DiMasi JA, Feldman L, Seckler A, Wilson A. Trends in risks associated with new drug development: success rates for investigational drugs. Clin. Pharm. Ther. 87(3), 272-277 (2010).

15. 2014 CMRInternational Pharmaceutical R\& D Factbook. CMR International, Thomson Reuters, Toronto, Canada (2014). http://citeseerx.ist.psu.edu/viewdoc/download?doi=10.1.1.675.2511\&rep=rep1\&type=pdf

16. Bailey J, Thew M, Balls M. Predicting human drug toxicity and safety via animal tests: can any one species predict drug toxicity in any other, and do monkeys help? Altern. Lab. Anim. 43(6), 393-403 (2015).

17. Greaves P, Williams A, Eve M. First dose of potential new medicines to humans: how animals help. Nat. Rev. Drug Disc. 3(3), 226 (2004).

18. Segall MD, Barber C. Addressing toxicity risk when designing and selecting compounds in early drug discovery. Drug Disc. Today 19(5), 688-693 (2014).

19. Ahuja V, Bokan S, Sharma S. Predicting toxicities in humans by nonclinical safety testing: an update with particular reference to anticancer compounds. Drug Disc. Today 22(1), 127-132 (2017).

20. Harley E. An analysis of the use of animals in predicting human toxicology and drug safety: a review. In: Understanding Animal Research. London, UK (2014). www.understandinganimalresearch.org.uk/files/7214/8458/1508/Bailey_analysis.pdf

21. Afonin S, Babii O, Reuter A et al. Light-controllable dithienylethene-modified cyclic peptides: photoswitching the in vivo toxicity in zebrafish embryos. Beilstein J. Org. Chem. 16, 39-49 (2020).

-. In vivo toxicity studies of photocontrollable peptidomimetics in zebrafish embryo.

22. Beharry AA, Wong L, Tropepe V, Woolley GA. Fluorescence imaging of azobenzene photoswitching in vivo. Angew. Chem. Int. Ed. 50(6), 1325-1327 (2011).

23. Rovira X, Trapero A, Pittolo $S$ et al. OptoGluNAM4.1, a photoswitchable allosteric antagonist for real-time control of mGlu4 receptor activity. Cell Chem. Biol. 23(8), 929-934 (2016).

-. In vivo demonstration of photopharmacology efficiency on zebrafish embryos and rodents. 
24. Gómez-Santacana X, Pittolo S, Rovira X et al. Illuminating phenylazopyridines to photoswitch metabotropic glutamate receptors: from the flask to the animals. ACS Cent. Sci. 3(1), 81-91 (2017).

25. Zussy C, Gómez-Santacana X, Rovira X et al. Dynamic modulation of inflammatory pain-related affective and sensory symptoms by optical control of amygdala metabotropic glutamate receptor 4. Mol. Psychiatry 23(3), 509-520 (2018).

26. Mehta ZB, Johnston NR, Nguyen-Tu MS et al. Remote control of glucose homeostasis in vivo using photopharmacology. Sci. Rep. 7(1), 291 (2017).

-. In vivo demonstration of photopharmacology efficiency and safety on rodents.

27. Schoenberger M, Damijonaitis A, Zhang Z, Nagel D, Trauner D. Development of a new photochromic ion channel blocker via azologization of fomocaine. ACS Chem. Neurosci. 5(7), 514-518 (2014).

28. Borowiak M, Nahaboo W, Reynders M et al. Photoswitchable inhibitors of microtubule dynamics optically control mitosis and cell death. Cell 162(2), 403-411 (2015).

29. Komarov IV, Afonin S, Babii O, Schober T, Ulrich AS. Efficiently photocontrollable or not? Biological activity of photoisomerizable diarylethenes. Chem. Eur. J. 24(44), 11245-11254 (2018).

30. Chen L, Zhu Y, Yang D, Zou R, Wu J, Tian H. Synthesis and antibacterial activities of antibacterial peptides with a spiropyran fluorescence probe. Sci. Rep. 4, 6860 (2014).

31. Petermayer C, Dube H. Indigoid photoswitches: visible light responsive molecular tools. Acc. Chem. Res. 51(5), 1153-1163 (2018).

32. Pianowski ZL. Recent implementations of molecular photoswitches into smart materials and biological systems. Chem. Eur. J. 25(20), 5128-5144 (2019).

33. Gause GF, Brazhnikova MG. Gramicidin S and its use in the treatment of infected wounds. Nature 154(3918), 703 (1944).

34. Global Peptide Therapeutics Market \& Clinical Pipeline Insight 2016. Research and Markets, OPNS Pharma, Dublin, Ireland (2016).

35. Albert L, Vázquez O. Photoswitchable peptides for spatiotemporal control of biological functions. Chem. Commun. 55, 10192-10213 (2019).

36. Antibiotic and Chemotherapy (6th Edition). Lambert HP, O'Grady FW (Eds). Churchill Livingstone, Edinburgh, UK (1992).

37. Okamoto K, Tomita Y, Yonezawa H, Hirohata T, Ogura R, Izumiya N. Inhibitory effect of gramicidin $S$ on the growth of murine tumor cells in vitro and in vivo. Oncology 41(1), 43-48 (1984).

38. Mosmann T. Rapid colorimetric assay for cellular growth and survival: application to proliferation and cytotoxicity assays. J. Immunol. Methods 65(1-2), 55-63 (1983).

39. Alley MC, Scudiero DA, Monks A et al. Feasibility of drug screening with panels of human tumor cell lines using a microculture tetrazolium assay. Cancer Res. 48(3), 589-601 (1988).

40. Enamine. Biology services (2019). https://enamine.net/discovery/biology-services

41. Animal Models in Toxicology (3rd Edition). Gad SC. (Ed.) CRC Press, FL, USA, 1152 (2015).

42. OECD. Guidance document on the recognition, assessment and use of clinical signs as humane end points for experimental animals used in safety evaluation, series on testing and assessment. Organisation for Economic Co-operation and Development, $\mathrm{N}^{\circ} 19$, ENV/JM/MONO (2000)7, Paris, France (2000). https://issuu.com/aaalac/docs/rr_humaneendpoints?e=6575905/2805072

43. Finney DJ. Probit Analysis (3rd Edition). 50-80 100-124 Cambridge University Press, Cambridge, UK (1971).

44. Hill JR. In vitro drug metabolism using liver microsomes. Curr. Protoc. Pharmacol. 23(1), 7.8.1-7.8.11 (2003).

45. Taglialatela M, Castaldo P, Pannaccione A, Giorgio G, Annunziato L. Human ether-a-gogo related gene (HERG) K channels as pharmacological targets: present and future implications. Biochem. Pharm. 55(11), 1741-1746 (1998).

46. Dhaneshwar S, Patil K, Bulbule M, Kinjawadekar V, Joshi D, Joshi V. Photodynamic therapy for cancer. Int. J. Pharm. Sci. Rev. Res. 27(2), 125-141 (2014).

47. Wilson BC, Patterson MS. The physics, biophysics and technology of photodynamic therapy. Phys. Med. Biol. 53 (9), R61 (2008).

48. Brown SB, Brown EA, Walker I. The present and future role of photodynamic therapy in cancer treatment. Lancet Oncol. 5(8), 497-508 (2004). 